\title{
Helmholtz dark solitons at nonlinear defocusing interfaces
}

\author{
Julio Sánchez Curto and Pedro Chamorro Posada \\ Departamento de Teoría de la Señal y Comunicaciones e Ingeniería Telemática, Universidad de Valladolid, ETSI \\ Telecomunicación, Campus Miguel Delibes s/n, Valladolid 47011, Spain \\ julsan@tel.uva.es \\ Graham S. McDonald \\ Joule Physics Laboratory, School of Computing, Science and Engineering, \\ University of Salford, Salford M5 4 WT, UK
}

\begin{abstract}
Dark soliton refraction at defocusing interfaces is analyzed within the framework of the noparaxial Helmholtz theory. A transition from internal to external refraction on varying the soliton grayness parameter is reported.

(C)2010 Optical Society of America

OCIS codes: $190.3270 ; 190.6135$
\end{abstract}

Spatial soliton refraction at the interface separating two nonlinear media has been traditionally studied in terms of the paraxial Nonlinear Schrödinger Equation (NSE) which limits the validity of the results to vanishingly small angles of incidence [1]. This restriction is released in the Helmholtz nonparaxial framework [2, 3] where the Nonlinear Helmholtz Equation (NHE) [4] has been proposed to describe the evolution of broad beams (when compared to the wavelength) propagating at arbitrary angles.

In our work, we analyze spatial soliton refraction at nonlinear interfaces using this Helmholtz theory $[5,6,7]$ which preserves the full inherent angular content of the problem. Such angular dependence has been captured in an unified generalized Snell's law [7]

$$
\gamma_{ \pm} n_{01} \cos \left(\theta_{n i}+\theta_{0 i}\right)=n_{02} \cos \left(\theta_{n t}+\theta_{0 t}\right)
$$

which describes the behavior of both bright and dark solitons evolving at nonlinear interfaces. In Eq. (1) $\theta_{n i}$ and $\theta_{n t}$ are the net angles of incidence and refraction, respectively, while $\theta_{0 i}$ and $\theta_{0 t}$ are the corresponding angular components associated to the intrinsic gray excitation of an incident [8] and a refracted [7] dark soliton. $\gamma_{ \pm}$is a nonlinear correction term [7]

$$
\gamma_{ \pm}=\left[\frac{\left(1+4 \kappa \beta_{ \pm}\right)}{1+4 \kappa \beta_{ \pm} \alpha_{2} n_{01} / \alpha_{1} n_{02}}\right]^{1 / 2}
$$

where the subscript \pm identifies the result for focusing $(+)[5,6]$ or defocusing $(-)$ [7] Kerr media. One has $\beta_{+}=\eta_{0}^{2} / 2$ for bright [5, 6] and $\beta_{-}=-u_{0}^{2}$ for black solitons [7], where $\eta_{0}$ and $u_{0}$ denote the amplitude and background amplitude of a bright and black soliton, respectively. In Eq. (2), $n_{0 i}$ and $\alpha_{i}$ account for the linear and nonlinear refractive indexes of the $i$ medium, respectively, and $\kappa$ is the nonparaxial parameter $[2,3]$.

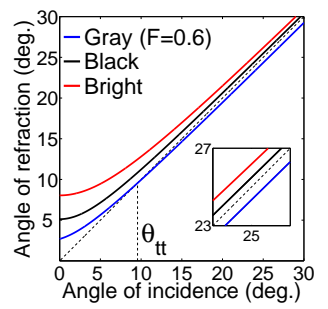

(a)

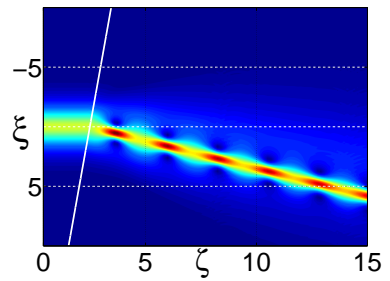

(b)

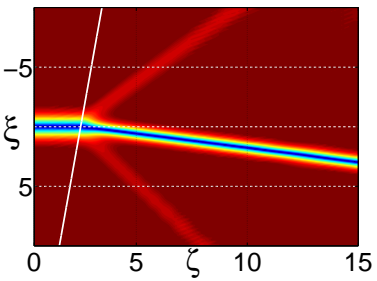

(c)

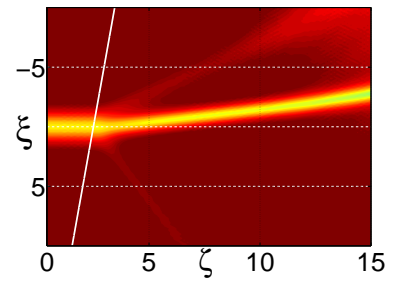

(d)

Figure 1: Generalized Snell's law for $n_{02}=1.008 n_{01}$ and $\alpha_{2}=3 \alpha_{1}$ (a). A bright (b), black (c) and a gray soliton with $F=0.6$ (d) incident at $25^{\circ}$ on the same interface. 
Equation (1) describes bright $\left(\gamma_{+}\right)$and black $\left(\gamma_{-}\right)$soliton refraction when $\theta_{0 i}=\theta_{0 t}=0$. For gray solitons, however, Eq. (1) has to be suplemented with the conservation of the soliton grayness [7]. Figure 1(a) shows Eq. (1) for a nonlinear interface with $n_{02}=1.008 n_{01}$ and $\alpha_{2}=3 \alpha_{1}$. While bright and black solitons undergo external refraction, a gray soliton may undergo either external or internal refraction depending on the angle of incidence. Such feature is captured in Figures 1(b), (c) and (d) which show the numerical results obtained for a bright, black and gray soliton, respectively, impinging on the same nonlinear interface at $25^{\circ}$. Instead of external refraction, the gray soliton undergoes internal refraction since the angle of incidence has been chosen according to the predictions shown in Figure 1 .

Gray soliton refraction is strongly dependent on the soliton grayness parameter $F$. This is captured in the results of Figure 2 which show the numerics of a gray soliton impinging on the same nonlinear interface $\left(n_{02}=1.0124 n_{01}\right.$ and $\left.\alpha_{2}=4 \alpha_{1}\right)$ at $30^{\circ}$ for soliton grayness parameter increasing from $F=0.2$ to $F=0.5$ in intervals of 0.1. Figures 2 (a), (b), (c) and (d) reveal how the net angle of refraction $\theta_{n t}$ decreases as $F$ grows, showing a transition in gray soliton refraction from external to internal.

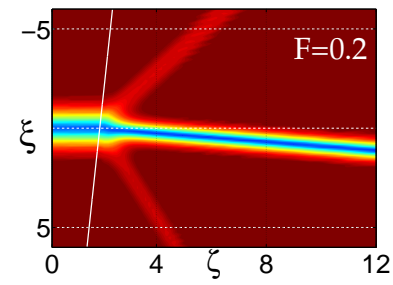

(a)

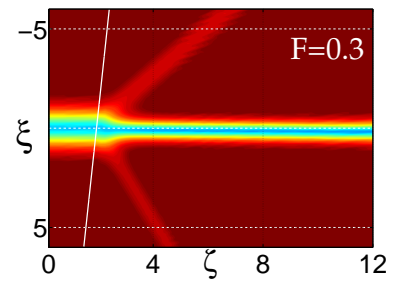

(b)

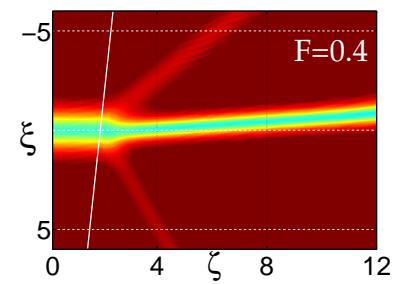

(c)

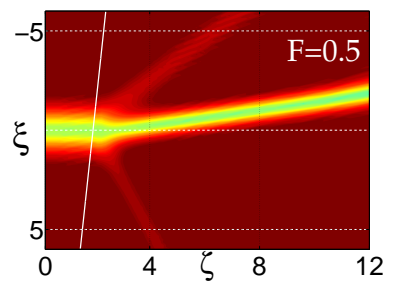

(d)

Figure 2: A gray soliton changing the net angle of refraction depending solely on the grayness parameter $F$.

This behaviour is completely ruled by (1). Larger values of $F$ increase the angular component associated to the intrinsic gray excitation of the refracted soliton $\theta_{0 t}$ [7], thus reducing the net angle of refraction $\theta_{n t}$.

In summary, we present a generalized Snell's law which addresses Helmholtz soliton refraction at the nonlinear interface separting both Kerr focusing and defocusing media. Developed within the framework of the NHE, its validity extends to arbitrary angles thus overcoming the angular restrictions inherent in the paraxial theory. We have also demonstrated that gray soliton refraction is governed by this law showing how a gray soliton can change from external to internal refraction on varying the soliton grayness parameter.

[1] A.B. Aceves, J.V. Moloney, and A.C. Newell, "Theory of light-beam propagation at nonlinear interfaces. I. Equivalentparticle theory for a single interface", Phys. Rev. A 39, 1809-1827 (1989).

[2] P. Chamorro-Posada, G.S. McDonald, and G.H.C. New, "Nonparaxial solitons," J. Mod. Opt. 45, 1111-1121 (1998).

[3] P. Chamorro-Posada, G.S. McDonald, and G.H.C. New, "Exact soliton solutions of the nonlinear Helmholtz equation," J. Opt. Soc. Am. B 19, 1216-1217 (2002).

[4] G. Fibich, "Small beam nonparaxiality arrests self-focusing of optical beams," Phys. Rev. Lett. 76, 4356-4359 (1996).

[5] J. Sánchez-Curto, P. Chamorro-Posada, and G.S. McDonald, "Helmholtz solitons at nonlinear interfaces," Opt. Lett. 32, 1126-1128 (2007).

[6] J. Sánchez-Curto, P. Chamorro-Posada, and G.S. McDonald, "Nonlinear interfaces: intrinsically nonparaxial regimes and effects," J. Opt. A: Pure Appl. Opt. 11, 054015 (2009).

[7] J. Sánchez-Curto, P. Chamorro-Posada, and G.S. McDonald, "Dark solitons at nonlinear interfaces," submitted.

[8] P. Chamorro-Posada and G.S. McDonald, "Helmholtz dark solitons," Opt. Lett. 28, 825-827 (2003). 